\title{
Helicobacter pylori: Interrelación de la prueba de urea en muestras de placa dental y biopsia gástrica
}

Helicobacter pylori: Interrelationship between the urea test in dental plaque samples and gastric biopsy

\begin{abstract}
Resumen
Con el objetivo de establecer la interrelación de la Prueba de urea en muestras de placa dental y la de biopsia gástrica para la determinación de la presencia del Helicobacter pylori; se realizó el estudio en 50 pacientes del Hospital Central de la Policía Nacional. Se tomaron simultáneamente muestras de placa dental y de biopsias gástricas en pacientes del servicio de Gastroenterología, a quienes se les indicó endoscopias por el médico tratante, obteniéndose muestras del estómago mediante sacabocado, las cuales fueron colocadas en caldo urea y transportadas al laboratorio del hospital para su incubación. Las muestras de placa dental fueron colocadas directamente en el caldo urea y llevados al laboratorio de Microbiología del la Facultad de Odontología de la Universidad Nacional Mayor de San Marcos para su incubación a $37^{\circ} \mathrm{C}$ y los resultados fueron leídos a las 24, 48 y 72 horas, y registrados en una base de datos. Los resultados de las biopsias gástricas fueron obtenidos del laboratorio de histopatología del Hospital. El análisis de los resultados obtenidos corrobora la hipótesis que señala que existe relación entre la determinación de la prueba de urea positiva en muestras de placa dental con las obtenidas en biopsias gástricas ya que se obtuvo un $68 \%$ de coincidencias de valores tanto positivos como negativos para ambos. La especificidad fue de $62 \%$ de los casos y la sensibilidad de $75 \%$; sin embargo, el nivel de interrelación no es elevado.
\end{abstract}

Palabras clave: Helicobacter pylori, Prueba de urea, Biopsia gástrica.

\begin{abstract}
With the purpose of establishing interrelationship between the urea test in dental plaque and gastric biopsy samples to determine the presence of Helicobacter pylori, this study was performed in 50 patients from the National Police Central Hospital. Simultaneously, samples from the dental plaque and gastric biopsies were taken from patients of the Gastroenterology Department, the same that were submitted to endoscopies by their medical attendant. Samples of their stomachs were obtained by a punching method, which were placed in urea broth and transported to the laboratory of the hospital for their incubation. The dental plaque samples were placed directly in the urea broth and taken to the Microbiology laboratory of the School of Dentistry of the San Marcos Major National University for their incubation at $37{ }^{\circ} \mathrm{C}$. The results were obtained 12, 48 and 72 hours later, and recorded into a database. The results from the gastric biopsies were obtained from the Histopathology laboratory of the Hospital. The analysis of the results obtained corroborates the hypothesis about the existence of a relationship between the determination of the positive urea test in dental plaque samples and the ones obtained from the gastric biopsies, since a 68 $\%$ coincidence of values was obtained, both positive and negatives. The specificity of the cases was of $62 \%$ and the sensibility of $75 \%$. However, the interrelationship level is not high.
\end{abstract}

Keywords: Helicobacter pylori, urea test, Gastric Biopsy.

\section{Introducción}

Helicobacter pylori (H.p) es un bacilo gramnegativo, curvado, móvil y microaerófilo resistente al medio ácido, que se encuentra en la mucosa gástrica del estómago asociado a diferentes enfermedades digestivas. La característica bioquímica más importante es la enzima ureasa, considerablemente más potente que la de otras bacterias. La infección por H.p es una de las más comunes en el hombre y aunque ocurre en todo el mundo, es más frecuente en los países en desarrollo y la prevalencia disminuye cuando aumenta el nivel socioeconómico. ${ }^{1}$ Es un patógeno que causa gastritis crónica, úlcera gástrica y duodenal, y en algunos casos contribuye al desarrollo de carcinoma gástrico, se asocia a linfoma asociado al tejido linfoide (MALT). ${ }^{2-3}$ La detección en sitios extraorales tales como la cavidad oral es aún controversial. ${ }^{4-10}$ Hay sugerencias que con protocolos usando PCR en la cavidad oral podría asumirse tener un rol de reservorio para reinfecciones. ${ }^{5,11-}$ ${ }^{13}$ En un estudio con pacientes diagnosticados con enfermedad gastroesofageo, se halló asociación significativa entre la presencia de H.p muestras de biopsia, placa dental y saliva en relacion con sujetos sin enfermedad y que no presentaban la bacteria. Igualmente, estudios de recuperacion por cultivos a partir de muestras de placa subgingival ${ }^{14}$ de-
Artículo Original

\section{Daniel De la Cruz Valle ${ }^{1}$ Hilda Moromi Nakata ${ }^{2}$}

${ }^{1}$ Cirujano Dentista, Práctica privada. Egresado de la Universidad Nacional Mayor de San Marcos

${ }^{2}$ Profesor principal del Departamento de Ciencias Básicas de la Facultad de Odontología de la Universidad Nacional Mayor de San Marcos

Tesis para optar el título de Cirujano Dentista

Correspondencia:

CD Daniel de la Cruz

Av. San Juan 285 zona B, San Juan de

Miraflores - Lima

Correo electrónico: daniel_dax@hotmail.com muestra su presencia; sin embargo, no se conoce si esta es solo transitoria o es un comensal permanente, igualmente se desconoce sobre el número necesario para producir reinfecciones. La variabilidad de los hallazgos igualmente depende de factores como los métodos y técnicas utilizadas y van de 1,6\% a $100 \% .^{14}$

La adquisición natural de H.p ocurre con frecuencia en la infancia y una vez que se establece, la infección persiste durante toda la vida, aunque también se ha descrito su eliminación natural. El mecanismo de adquisición es por contacto interpersonal, animales o con agua contaminada. Se estima que en los 
países en desarrollo, la mayoría de las personas (el $80 \%$, aproximadamente) se infecta con H.p a una edad promedio de 10 ańos; ${ }^{15-16}$ en un estudio realizado en jóvenes de 15-24 años, se detectó una seroprevalencia de $72,5 \%{ }^{10}$ Para diagnosticar la infección por H.p se pueden realizar métodos invasivos (requieren endoscopia con toma de biopsia gástrica) o métodos no invasivos (no requieren endoscopia previa). Los métodos no invasivos incluyen la prueba del aliento de urea, pruebas serológicas, y detección de antígenos en materia fecal. El test del aliento de la urea se basa en la actividad de la ureasa derivada del H. pylori en el estómago; detectando cualitativamente infección activa con una sensibilidad y especificidad de más del $90 \mathrm{~m} \%$. Esta prueba está indicada para el diagnóstico inicial de la infección y para el seguimiento luego de realizado un tratamiento de erradicación. ${ }^{15}$ A la hora de elegir uno u otro método, hay que tener en cuenta el objetivo del diagnóstico (epidemiológico, diagnóstico o de seguimiento), la experiencia del personal y disponibilidad de medios; las características del paciente (prevalencia de $H$. pylori en la población, edad del paciente, medicación previa, etc.). No se debe olvidar que mientras que todos los métodos pueden servir para diagnosticar la infección por H.p (con diferentes porcentajes de sensibilidad y especificidad), la endoscopia con toma de biopsia para estudio histológico permite, además, diagnosticar el tipo de enfermedad. H. pylori posee una ureasa que le capacita para la colonización y persistencia en la cavidad gástrica. La potencia de la ureasa es muy superior a la de otras bacterias, incluida Proteus sp. La enzima cumple tres funciones principales: protección frente al ácido de la mucosa gástrica, provisión de nitrógeno en forma de amonio y como factor de virulencia en la patogenia de la úlcera gástrica.

El fundamento de la prueba rápida de la ureasa consiste en detectar la presencia de la enzima en la que el $H$. pylori descompone la urea en anhídrido carbónico y amoniaco, lo cual genera un $\mathrm{pH}$ básico que va a ponerse en evidencia mediante el cambio de color del medio de naranja-ladrillo a rosa grosella debido al viraje del indicador de $\mathrm{pH}$.

La prueba de la ureasa rápida se puede realizar directamente con la muestra de biopsia gástrica, obtenida mediante endoscopia digestiva alta. Se recomiendan dos biopsias, una de cuerpo y otra de antro para el diagnóstico. Las muestras pueden ser inoculadas en la misma sala de endoscopias por lo que no necesitan ningún medio de transporte. En general, son sistemas comerciales muy sencillos de utilizar y los resultados se interpretan en un intervalo corto de tiempo (media hora), observando el cambio en el color del reactivo. Los resultados de sensibilidad y especificidad son, en general, superiores al $80 \%$ y $90 \%$, respectivamente.

También se puede utilizar una solución preparada en el laboratorio que contenga urea al 3-4 \% e indicador de $\mathrm{pH}$; sin embargo, los resultados de sensibilidad pueden ser algo menores. En cualquier caso, debería cubrirse distintos tiempos de incubación (24 horas a 7-14 días), las necesidades nutricionales y respiratorias con atmósferas aerobias y anaerobias y con $5 \%$ de $\mathrm{CO} 2 .{ }^{17-18}$

El objetivo del presente estudio fue establecer la relación de la aplicación de la prueba de urea para Helicobacter pylori en muestras de placa dental y de biopsia gástrica.

\section{Material y método}

El tipo de investigación es analítico y transversal. Las muestras fueron obtenidas de 50 pacientes de ambos sexos del servicio de Gastroenterología del Hospital Central de la Policía Nacional, en Lima; con el debido consentimiento informado, y previo registro de datos del paciente.

\section{Toma de muestra de placa dental}

Inicialmente, se aisló parcialmente la pieza dental con rollos de algodón estériles para evitar la contaminación de la zona circundante.

Tabla 1. Prueba de la urea en muestras de placa dental y biopsia, según pacientes y sexo en el Servicio de Gastroenterología del Hospital central de la Policía Nacional. Lima, 2009

\begin{tabular}{lcccc}
\hline PACIENTE & \multicolumn{2}{c}{ MUESTRA DE PLACA $(\mathrm{n}=38)$} & \multicolumn{2}{c}{ MUESTRA DE BIOPSIA $(\mathrm{n}=82)$} \\
\hline & NEGATIVO & POSITIVO & NEGATIVO & POSITIVO \\
Femenino & 11 & 11 & 14 & 8 \\
Masculino & 11 & 17 & 12 & 16 \\
Total & 22 & 28 & 26 & 24 \\
$\%$ & 44 & 56 & 52 & 48 \\
\hline
\end{tabular}

Se obtuvo mediante curetas estériles, cantidad suficiente y representativa de la muestra en estudio.

Luego, se realizó la toma de muestra en el momento que se ejecutó la endoscopia gástrica y la toma la biopsia.

Las muestras de placa bacteriana se colocaron directamente en el caldo urea debidamente rotulado con los datos correspondientes.

El transporte se realizó durante las primeras 2 horas después de la toma de la muestra

Las muestras llegadas al laboratorio en el tiempo establecido, se incubaron a $37^{\circ} \mathrm{C}$ y las lecturas se realizaron a las 24, 48 y 72 horas, observándose los cambios de color del medio a un color grosella.

\section{Toma de muestra para biopsia}

Se realizaron en el servicio de Gastroenterología simultáneamente a la toma de placa dental y por personal médico especializado, a primera hora de atención (08:00 a.m.). Se colocaron en frascos rotulados con el nombre, número de historia clínica y se procesaron el laboratorio del Hospital. Las 50 biopsias gástricas obtenidas se separaron cuidadosamente de las pinzas para biopsia mediante el uso de agujas hipodérmicas y fueron montadas sobre papel de filtro, las biopsias se orientaron sobre el papel con la mucosa hacia arriba y se separaron entre ellas de 3 a $4 \mathrm{~mm}$. Inmediatamente, se fijaron en formalina taponada al $10 \%$ en frascos previamente rotulados con los datos del paciente (nombre, sexo, edad, código de historia clínica) y permanecieron en fijación durante $12 \mathrm{a}$ 36 horas. Posteriormente, se montaron en bloques de parafina, se seccionaron (3-4 $\mu \mathrm{m})$ y se colorearon con hematoxilina eosina y sometidos a la prueba de la 
urea colocando Urepil-II como reactivo y en el caso de la biopsia, se aprovecha la formación de amonio y viendo el cambio de coloración.

\section{Resultados}

Las muestras Placa dental y de biopsia gástrica de los 50 pacientes correspondió: $22(44 \%)$ a pacientes masculinos y $28(56 \%)$ a femeninos. Las edades fluctuaron entre los 20 - 90 años.

Tabla 2. Prueba de Urea en muestras de Placa dental y Biopsias en el Hospital de Policía de Lima, 2009

\begin{tabular}{lcccc}
\hline MUESTRA DE PLACA DENTAL & MUESTRA DE BIOPSIA & & TOTAL & $\%$ \\
\hline & NEGATIVO & POSITIVO & & \\
NEGATIVO & 16 & 6 & 22 & 54 \\
POSITIVO & 10 & 18 & 28 & \\
Total & 26 & 24 & 50 & 100 \\
$\%$ & 52 & 48 & & \\
\hline
\end{tabular}

En la Tabla 1, se observa los resultados de la Prueba de urea en las muestras de placa dental y biopsia gástrica donde el $56 \%$ de las muestras dieron positivos (Fig. 1) para Placa dental y el $48 \%$ para las muestras de Biopsia.

El análisis y los resultados de la relación entre las muestras de los pacientes, se observan en la Tabla 2.

En 6/24 casos de biopsia positiva, no se detectó en placa dental, es decir, en el $25 \%$ falso negativo.

En 10/26 casos se detecto en placa pero no en biopsia, podría ser portador en placa o falso positivo, equivale al $38 \%$.

En el $62 \%$ de los casos (16/26) resultaron coincidentemente Negativos (especificidad).

El $75 \%(18 / 24)$ resultaron positivos (sensibilidad).

Coincidencias positivas (18), más negativos (16), dan 34/50, lo que significa $68 \%$ correspondencia, el valor predic-

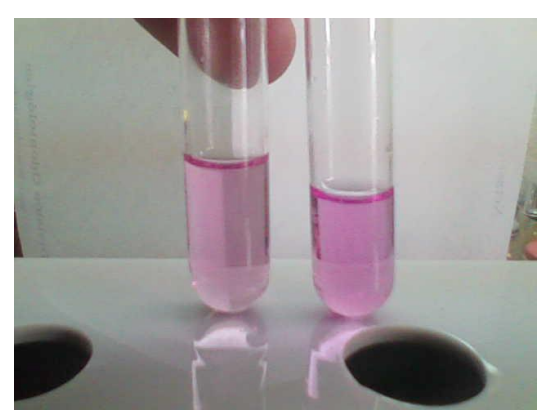

Fig 1. Prueba de la urea positiva. tivo positivo del $64 \%$ y predictivo negativo del $72 \%$.

\section{Discusión}

De acuerdo a algunos autores, la presencia del Helicobacter pylori en cavidad oral es bastante marcada según el tipo de población como lo indica Fuenmayor y cols. ${ }^{19}$ ya que en su estudio, 35 pacientes fueron sometidos a una prueba similar a esta como la prueba de ureasa

no comercial y de bajo costo, preparada con agar urea de Christensen, aplicada a biopsias gástricas antrales de 75 adultos sintomáticos, sometidos a endoscopia gastrointestinal de los cuales un 94,6 $\%$ fue correctamente diagnosticado, el comportamiento de la prueba de ureasa ensayada sugiere que esta es una herramienta altamente confiable y de bajo costo para el diagnóstico de la infección por $H$. pylori en individuos sometidos a endoscopia gastrointestinal.

Según el trabajo de Scarano Pereira G. $A$, realizado en $B_{r a s i l}{ }^{20}$, se comparó placa dental con biopsias gástricas obteniéndose que la presencia de $H$. pylori fue observada en el $100 \%$ de las muestras de placa dental y en el 66,67 \% de las de mucosa gástrica. De los 48 pacientes, se constató que $42(87,5 \%)$ presentaban alteraciones gastroduodenales $y$, entre ellos, 29 (69,05\%) presentaron reacción positiva para Helicobacter pylori en placa dental y en mucosa gástrica. Entre los 6 pacientes con endoscopia normal, se encontraron 3 casos $(50 \%)$ con presencia simultánea de $H$. pylori en placa dental y mucosa gástrica. La diferencia con los resultados del presente estudio da una positividad en placa de $56 \%$ y en biospsia de $48 \%$. Los resultados sugieren la presencia simultánea de $H$. pylori en placa dental y mucosa gástrica en elevadas proporciones. Nuestro estudio halló coincidencias entre positivas y negativas en un $68 \%$. Estos resultados permiten sugerir, además, que la placa dental puede constituir un reservorio importante de este microorganismo como también lo indica, Moromi y cols., ${ }^{14}$ en un estudio realizado en el año 2001, en la Facultad de Odontología de la UNMSM, en el cual se determino la prevalencia de $H$. pylori en pacientes con gingivitis y enfermedad periodontal, donde se recuperó mediante cultivo, Helicobacter pylori en 9,4 \% de la población mayormente en el grupo etario de 20 a 30 ańos y más frecuentemente en mujeres que hombres, como el presente trabajo que obtuvo mayor presencia en mujeres y con el grupos etarios de 41 a 70 años. Otros estudios también han relacionado la cavidad oral como reservorio, como lo indican los estudios realizados en el Hospital Angamos EsSalud, ${ }^{21}$ en el Hospital Universitario de Caracas, Venezuela ${ }^{22}$ y en el Hospital Clínico Universitario de la Universidad Central de Venezuela ${ }^{23}$ con resultados donde la placa dental es un reservorio para esta bacteria Helicobacter pylori.

Tamariz Ortiz y cols. ${ }^{24}$ obtuvieron cepas de Helicobacter pylori aisladas de biopsias gástricas en las placas de cultivo. Las colonias obtenidas fueron identificadas mediante coloración Gram, prueba oxidasa, catalasa y ureasa, siendo positivas a todas ellas lo que nos dice que la prueba de la ureasa funciona muy bien para grupos de estudio, como también otros estudios realizados con otras técnicas como las prueba de aliento marcan un punto importante en el diagnóstico de estos tipos de pruebas para la identificación del $H$. pylori. Los valores obtenidos tanto en las muestras de placa dental como en las muestras gástricas reflejan una relación estrecha de condiciones, lo que contribuye a una presencia la bacteria Helicobacter pylori, por lo que se puede demostrar el crecimiento de esta bacteria mediante la prueba de urea y así poder apoyar en el diagnóstico para poder tener un mejor manejo preventivo en beneficio de los pacientes.

La prueba de urea por la toma de muestra de placa dental no es invasiva por lo que para el paciente es menos traumático que la endoscopia y esta prueba la podemos hacer en el consultorio en coordinación con el médico especialista, además que se puede aplicar a grandes masas de pacientes y la necesidad de hacer la endoscopia, rectificaría en todo caso los hallazgos.

Esta prueba endoscópica es muchas veces vital para la identificación de enfermedades gastrointestinales como gastritis, úlcera péptica, pólipos y cáncer, que a su vez los odontólogos pueden orientar con una prueba sencilla y ayudar así al beneficio del paciente. 
Por tanto, podemos inferir que la placa dental representa un adecuado reservorio para la presencia de Helicobacter pylori como en el presente estudio de donde se obtuvo muestra de la población del lugar y procedencia que podría presentarse como un referente. Un punto importante es el relacionado con las condiciones higiénico-sanitarias y con un bajo nivel de vida (hacinamiento en las viviendas, camas compartidas, etc.); además, los valores de prevalencia son más elevados en las etapas más avanzadas de la vida como representa los resultados de este estudio.

\section{Conclusiones}

De acuerdo con los resultados encontrados en el estudio, se puede concluir que considerando que a pesar de existir coincidencias de resultados positivos y negativos simultáneas en un $68 \%$ de las muestras de placa dental y biopsia, habiendo hallado una especificidad de $62 \%$ y sensibilidad para el $75 \%$, el nivel de interrelación no es elevado.

\section{Referencias bibliográficas}

1. López-Brea M, Alarcón T, Baquero M, Domingo D, López-Brea $M$, Royo G Diagnóstico microbiológico de la infección por Helicobacter pylori. Enfermedades Infecciosas y Microbiología Clínica 2004. http:// www.seimc.org/documentos/protocolos/microbiologia/cap17.htm (acceso octubre 2009).

2. Atherton JC, Cao P, Peek RM Jr, Tummuru MKR, Blaser MJ, Cover TL. Mosaicism in vacuolatin cytotoxin alleles of Helicobacter pylori. Association of specific vacA types with cytotoxin production and peptic ulceration. J Biol Chem 1995; 270: 1771-1777.

3. Cover TL, Blaser MJ. Helicobacter pylori: a bacterial cause of gastritis, peptic ulcer disease and gastric cancer. ASM News 1995; 61: 21-26.

4. Butt AK, Khan AA, Izhar M, Alam A, Shah SW, Shafqat F. Correlation of Helicobacter pylori in dental plaque and gastric mucosa of dyspeptic patients. J Pak Med Assoc 2002; 52: 196-200.

5. Dowsett SA, Kowolik MJ. Oral Helicobacter pylori: can we stomac h it? Crit Rev Oral Biol Med 2003; 14: 226-233.
6. Goosen C, Theron J, Ntsala M et al. Evaluation of a novel heminested PCR assay based on the phosphoglucosamine mutase gene for detection of Helicobacter pylori in saliva and dental plaque. J Clin Microbiol 2002; 40: 205-209.

7. Kabir S. Detection of Helicobacter pylori DNA in feces and saliva by polymerase chain reaction: a review. Helicobacter 2004; 9: 115-123.

8. Luman W, Alkout AM, Blackwell CC, Weir DM, Plamer KR. Helicobacter pylori in the mouth-negative isolation from dental plaque and saliva. Eur J Gastroenterol Hepatol 1996; 8: 1-2.

9. Majmudar P, Shah SM, Dhunjibhoy KR, Desai HG. Isolation of Helicobacter pylori from dental plaques in healthy volunteers. Indian J Gastroenterol 1990; 10: 33-34.

10. Moromi NH, Calle ES, Martinez CE, Villavicencio GJ, Zambrano DS. Prevalencia del Helicobacter pylori mediante ELISA en estudiantes de la Facultad de odontología de la UNMSM. Odont. Sanmarquina 2002; 1(9): 6-10.

11. Desai HG, Gill HH, Shankaran K, Metha PR, Prabhu SR. Dental plaque: a permanent reservoir of Helicobacter pylori? Scand J Gastroenterol 1991; 26: 1205-1208.

12. Pytko-Polonczyk J, Konturek SJ, Karczewska E, Bielanski W, Kaczmarczyk-Stachowska A. Oral cavity as permanent reservoir of Helicobacter pylori and potential source of reinfection. J Physiol Pharmacol 1996; 47: 121-129.

13. Morales-Espinosa R, FernandezPresas A, Gonzalez-Valencia, y col. Helicobacter pylori in the oral cavity is associated with gastroesophageal disease. Oral Microbiol Immunol 2009; 24: 464-468.

14 Moromi NH, Calle ES y Zambrano de la Peńa S. Prevalencia de H.p en paciente con Enfermedad periodontal. Odontol Sanmarquina 2001: 1(7): 23-26.

15 Sullivan PB, Thomas JE, Wight DGD et al. Helicobacter pylori in Gambian children with chronic diarrhea and malnutrition. Arch Dis Child 1990; 65: 189-191.

16 Sainz R, Borda F, Domínguez E et al. Conferencia española de consenso sobre la infección por Helico- bacter pylori. Rev Esp Enferm Dig 1999; 91:777- 784.

17 J. Liébana Ureña, Microbiología Oral. Ed. McGraw-Hill Interamericana 1997. p. 431-444

18 Moromi Hilda. Microbiología periodontal: Pruebas diagnósticas, Odontología Sanmarquina 2003; 6(11): 43-47.

19 Fuenmayor B., Alisbeth; Hernández R., Ileana; Paz M., América; Cavazza, María E. y Lizarzábal G., Maribel Nuevas evidencias sobre la utilidad diagnóstica de una fórmula no comercial para la detección de la actividad de ureasa de Helicobacter pylori en biopsias gástricas Kasmera 34(1): 40 - 52, enero - junio 2006 ISSN 00755222 / Depósito legal $196202 Z U 39$.

20 Giuseppe Anacleto Scarano Pereira, Arnaldo Correia de Medeiros, Maria Sueli Marques Soares, Eduardo Chimenos Küstner, Rosimar de Castro Barreto, Mónica Perdomo Lovera Detección de Helicobacter pylori en placa dental y en mucosa gástrica de pacientes sometidos a endoscopia digestiva. Acta odontol. Venezuela v. 43 n.2 Caracas mayo 2005.

21. Chumpitaz CJ, Gutiérrez MJ, Córdova AR, Sánchez MM, Vásquez $\mathrm{VN}$ et al. Aislamiento de Helicobacter pylori en sarro dental de pacientes con gastritis del policlínico "Angamos". Rev. Gastroenterol. 2006; 26 (4).

22 Berroteran A, Perrone M, Correnti M, Cavazza ME, Lecuna V, López T, Avila M. La placa dental como reservorio de Helicobacter pylori. Rev. Soc. Ven. Microbiol. 2007; 27(2).

23 Berroteran A, Perrone M, Correnti M, Cavazza ME, Tombazzi, C, Lecuna V, Goncalvez R, Prevalencia de Helicobacter pylori en el estómago y placa dental de una muestra de la población en Venezuela. Acta Odontol. Venez. 2001; 39(2): 35-41.

24. Tamariz Ortiz, Jesús Humberto; Capcha Mendoza, Roberto; Palomino Cadenas, Edwin Julio et al. Actividad antibacteriana de la Sangre de Grado (Croton lechleri) frente al Helicobacter pylori. Rev Med Hered, abr. 2003, 14(2): 81-88.

Fecha de recepción: 5-5-10

Fecha de aprobación: 10-06-10 